\title{
Isolated Non-Resolving Vulvar Lesion as a Presentation of Disseminated Histoplasmosis in a Woman with HIV
}

\author{
Moni Roy ${ }^{1}$, Ashish Kumar Roy ${ }^{1}$, Jan Nalinee Upalakalin², Sharjeel Ahmad ${ }^{3}$ \\ ${ }^{1}$ Department of Internal Medicine, University of Illinois College of Medicine, OSF Saint Francis Medical Center, Peoria, Illinois, USA \\ ${ }^{2}$ Department of Pathology, OSF Saint Francis Medical Center, Peoria, Illinois, USA \\ ${ }^{3}$ Department of Internal Medicine, Section of Infectious Diseases, University of Illinois College of Medicine, Peoria, Illinois, USA
}

Received: 15/06/2020

Accepted: $19 / 06 / 2020$

Published: $16 / 07 / 2020 x$

How to cite this article: Roy M, Roy AK, Upalakalin, JN Ahmad S. Isolated non-resolving vulvar lesion as a presentation of disseminated histoplasmosis in a woman with HIV. EJCRIM 2020;7: doi:10.12890/2020_001814.

Conflicts of Interests: The Authors declare that there are no competing interests.

This article is licensed under a Commons Attribution Non-Commercial 4.0 License

\section{ABSTRACT}

Disseminated histoplasmosis is most commonly caused by Histoplasma capsulatum and is a known opportunistic infection in immunocompromised patients. The clinical presentation of histoplasmosis varies from asymptomatic to a progressive disseminated form. Pulmonary and CNS involvement is common in disseminated histoplasmosis. Rarely, disseminated disease can present as vulvar lesion in the absence of pulmonary symptoms, causing a delay in diagnosis. As per our PubMed literature search, there have only been two reported cases where vulvar lesion was the only presenting symptom of disseminated disease. In our patient, a histopathological diagnosis was made with staining showing budding yeast forms of histoplasma.

\section{LEARNING POINTS}

- Histoplasmosis can be a cause of isolated non-resolving vulvar lesion in immunocompromised patients.

- In patients at risk, we recommend work-up for disseminated histoplasmosis with urine histoplasma antigen and further imaging to rule out disseminated infection when histoplasma is identified on local biopsy samples.

- In patients from endemic regions and in non-resolving vulvar lesions, fungal infection should be considered.

\section{KEYWORDS}

Disseminated histoplasmosis, vulvar lesion, HIV/AIDS

\section{CASE DESCRIPTION}

A 36-year-old medically non-compliant woman with a history of acquired immunodeficiency syndrome (AIDS) and inconsistent use of highly active antiretroviral therapy (HAART) was diagnosed with Bartholin's abscess over the left labia. The abscess drained on its own and the patient was prescribed a 7-day course of trimethoprim-sulfamethoxazole. The wound did not heal completely after initial drainage. The patient denied fevers, chills, nausea, vomiting or shortness of breath. She underwent left labial examination under anaesthesia with labial biopsies due to concern for malignancy. Operative findings showed denuded and ulcerated vaginal epithelium at the posterior fourchette and bilateral lower labia majora. Malodorous drainage from the wound was seen with no fistula noted.

While histopathology results were pending, the patient's pain worsened after biopsy and she was admitted for intravenous antibiotics for suspected superimposed bacterial infection. Treatment with vancomycin and piperacillin-tazobactam was started. Azithromycin once 
weekly was added for Mycobacterium avium prophylaxis and also to provide coverage against Haemophilus ducreyi. The differential diagnosis included syphilis, fungal infection, pyoderma gangrenosum, mycobacterial infection, chancroid and malignancy.

Methods and procedures

The white cell count was $4.54 \times 10^{9}$ cells/l, with a low lymphocyte and elevated neutrophil differential. The absolute CD4 count was 97 cells/ $\mu \mathrm{l}$ with a detectable viral load of 108 copies/ $\mu$ l. The biopsy showed ulcerated squamous mucosa with granulation tissue in the absence of any malignant cells. Acid-fast bacilli stain, Grocott's methenamine silver stain (GMS) and periodic acid-Schiff stain (PAS) were applied to samples, with the GMS stain showing budding yeast, consistent with Histoplasma species (Fig. 1). The histoplasma antigen level in urine was elevated at $3.09 \mathrm{ng} / \mathrm{ml}$ (>0.5 considered positive), histoplasma fungal antibody was negative, and blastomyces urine antigen was elevated at $4.8 \mathrm{ng} / \mathrm{ml}$ (normal range, $0.2-14.7$ ), suspected to be due to histoplasma cross-reactivity. Coccidioidomycosis antibody was negative and Cryptococcus neoformans antigen level was not detected. Blood cultures for bacteria, mycobacteria and fungus remained negative.

Computed tomography (CT) of the abdomen and pelvis showed slight thickening and heterogeneity of labial tissue without fluid collection or abscess. Mild splenomegaly and inguinal lymphadenopathy were noted (Fig. 2). CT of the chest showed no nodules or any mediastinal or hilar lymphadenopathy. A chest $\mathrm{x}$-ray showed no acute process (Fig. 3).

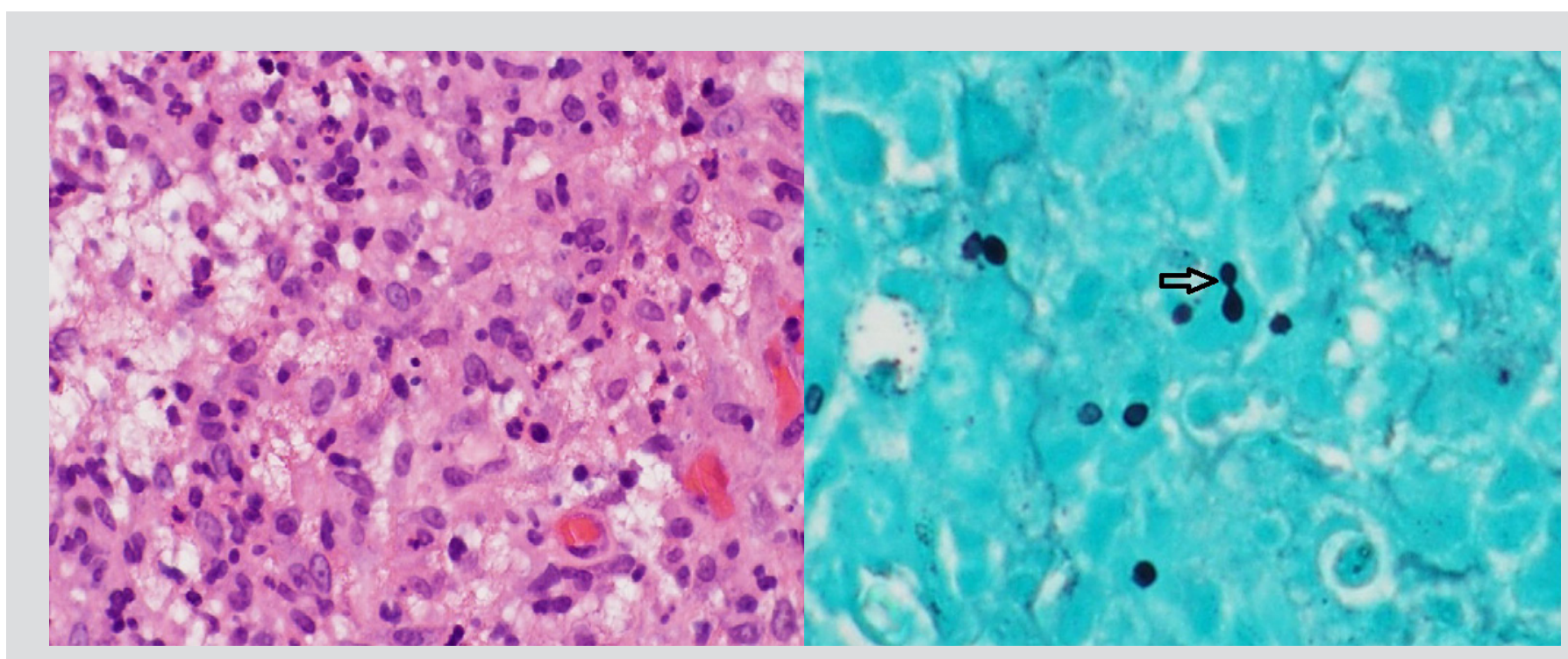

Figure 1. Histopathology of the vulvar lesion demonstrating atypical granulomatous inflammation on haematoxylin and eosin stain (400x magnification) and budding yeast shown by a right arrow on Grocott's methenamine silver stain (400x magnification)
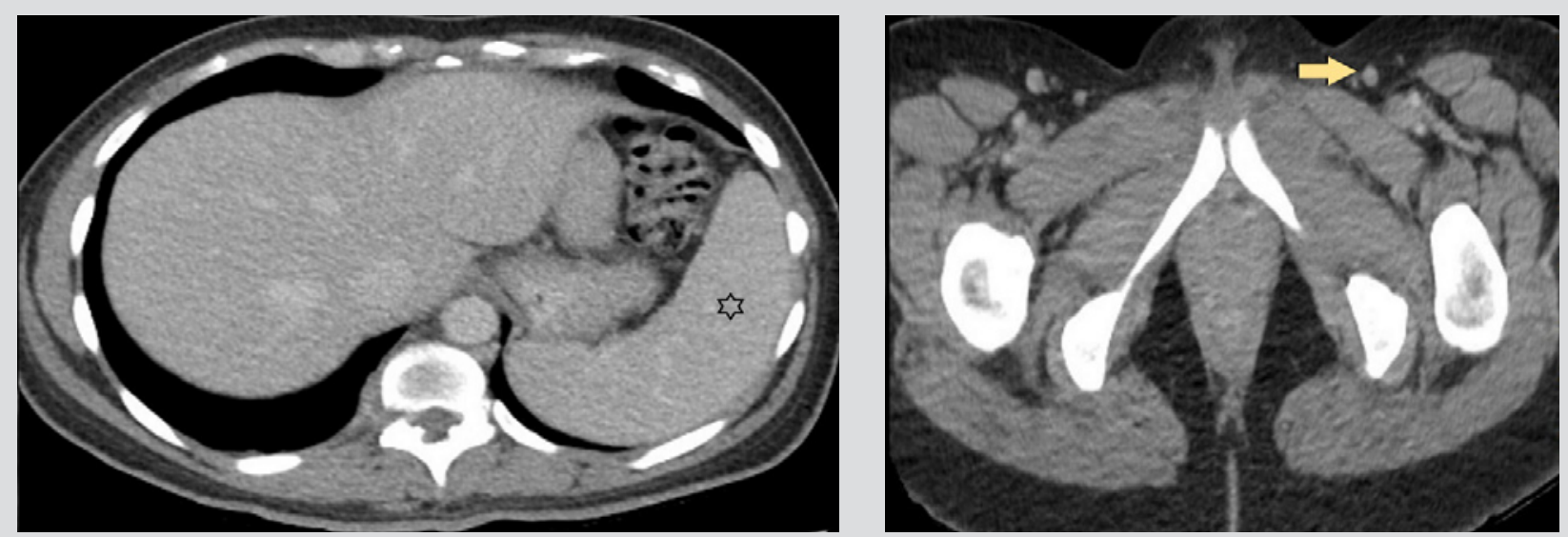

Figure 2. CT of the abdomen and pelvis showing mild splenomegaly (marked by a star) and inguinal lymphadenopathy (marked by a right arrow). 
After budding yeast were noted on fungal stain, the patient was started on intravenous amphotericin B for 2 weeks and then switched to oral itraconazole. Vancomycin and piperacillin-tazobactam were discontinued. The patient was also started on dapsone for Pneumocystis jiroveci pneumonia (PJP) prophylaxis. Unfortunately, the patient was lost to follow-up after discharge from the hospital.
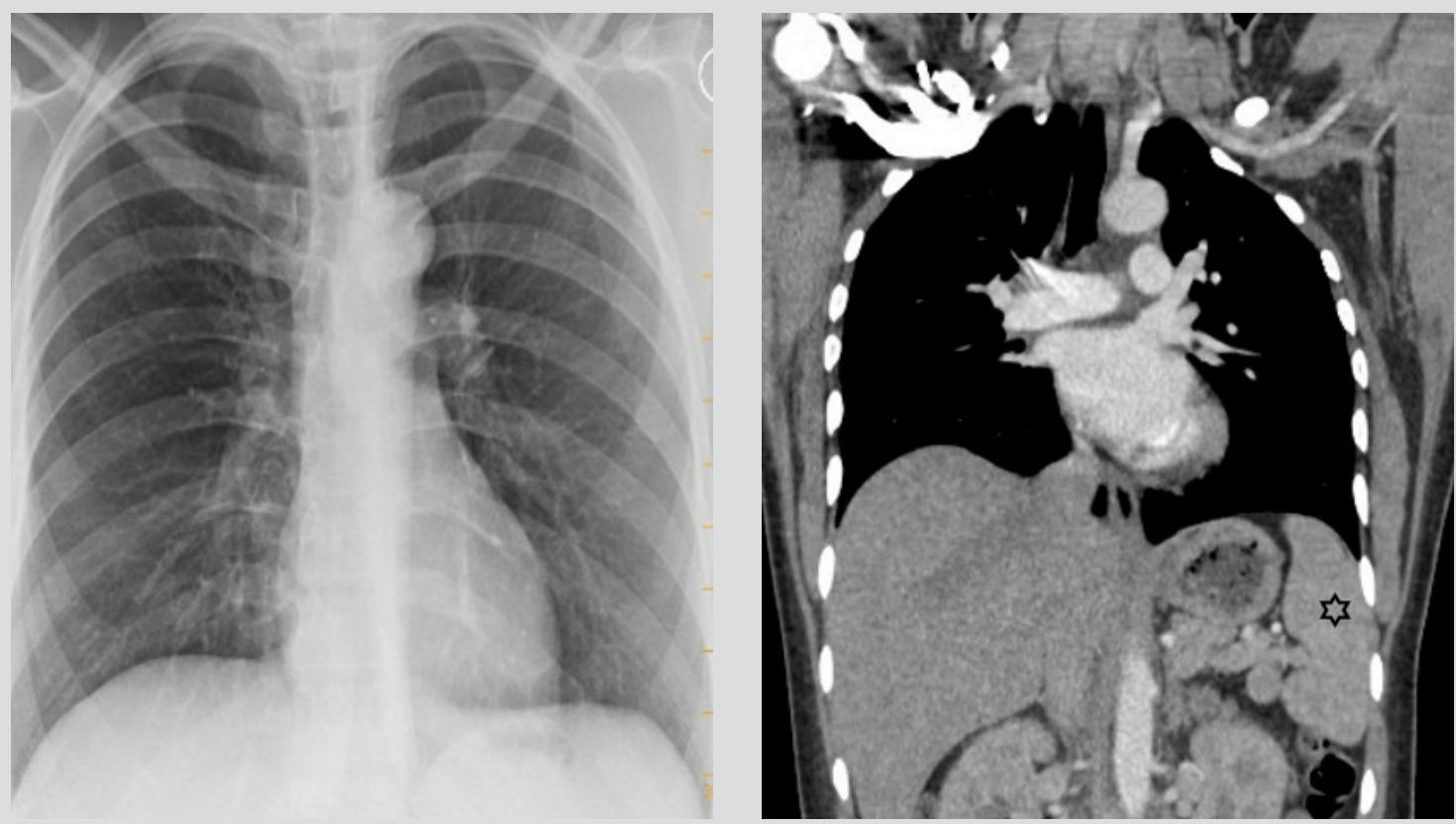

Figure 3. Chest $x$-ray and CT of the chest showing normal findings. Splenomegaly is also noted on the coronal CT of the chest (marked by a star).

\section{DISCUSSION}

Histoplasmosis in immunocompetent adults is usually subclinical, but disseminated histoplasmosis is frequently encountered in immunocompromised hosts, presenting with systemic manifestations of fever, weight loss and pulmonary symptoms. Isolated genital lesion as the only presentation of disseminated infection is a very rare finding. There have been only two case reports of vulvar lesion as the presenting symptom of disseminated infection in females ${ }^{[1,2]}$. Smith et al. reported the case of 63-year-old woman presenting with multiple genital lesions who was diagnosed by Papanicolaou and Giemsa staining of vulvar smears and then treated with ketoconazole ${ }^{[1]}$. In 2013, Shine et al. reported the second case of vulvar lesion without pulmonary involvement in a woman with AIDS ${ }^{[2]}$. Non-resolving vulvar lesions in adults raise concern for malignancy and a biopsy should be obtained early. Urogenital infections due to histoplasma have been reported in autopsy findings of disseminated histoplasmosis in males and females. It most commonly involved the kidney and most of these cases remained asymptomatic ${ }^{[3]}$. Isolated genital involvement in males has been reported by some authors and may present as phimosis or epididymitis and rarely can mimic Fournier's gangrene ${ }^{[4-6]}$. In patients with inconclusive results for malignancy, a low threshold should be maintained for fungal staining of the sample. In case of detection of histoplasma on histopathology, we recommend further work-up to rule out disseminated infection. As demonstrated in our patient, histopathology led to the final diagnosis. Further work-up showed positive urine histoplasma antigen and imaging study showed mild splenomegaly and inguinal lymphadenopathy, suggestive of more widespread infection.

\section{REFERENCES}

1. Smith MB, Schnadig VJ, Zaharopoulos P, Van Hook C. Disseminated Histoplasma capsulatum infection presenting as genital ulcerations. Obstet Gynecol 1997;89(5 Pt 2):842-844

2. Shine MM, Shah MM, Hanna CA, Bevis KS. Vulvar histoplasmosis as a rare cause of genital ulceration. Obstet Gynecol 2013;122(2 Pt 2):449-452.

3. Friskel E, Klotz SA, Bartholomew W, Dixon A. Two unusual presentations of urogenital histoplasmosis and a review of the literature. Clin Infect Dis 2000;31(1):189-191.

4. Randhawa HS, Chaturvedi S, Khan ZU, Chaturvedi VP, Jain SK, Jain RC, et al. Epididymal histoplasmosis diagnosed by isolation of Histoplasma capsulatum from semen. Mycopathologia 1995;131(3):173-177.

5. Kauffman CA, Slama TG, Wheat LJ. Histoplasma capsulatum epididymitis. J Urol 1981;125(3):434-435.

6. Sachdev R, Goel RK, Malviya S, Goel S, Gajendra S. Scrotal histoplasmosis masquerading as Fournier's gangrene. Int J Surg Pathol 2017;25(6):509-510. 